\title{
Electronic immunization information systems: a case report of lessons learned from implementation in Pakistan
}

\author{
Erin Sullivan ${ }^{1}$, Tariq Masood ${ }^{2}$, Wajiha Javed ${ }^{1}$, Katharine Bagshaw ${ }^{2}$, Steve Ollis ${ }^{2}$, Presha Regmi ${ }^{1}$, \\ Syed Muhammad Akbar Gardezi ${ }^{1}$ \\ ${ }^{1}$ Jhpiego, Baltimore, MD, USA; ${ }^{2}$ John Snow, Inc., Arlington, VA, USA \\ Correspondence to: Erin Sullivan. Jhpiego, 1615 Thames St \#200, Baltimore, MD 21231, USA. Email: esullie@gmail.com.
}

\begin{abstract}
Pakistan ranks third globally in the number of unvaccinated and under-vaccinated children, and Sindh province has one of the lowest vaccination rates in the country. Electronic census-based immunization information systems have the potential to bolster traditional immunization service delivery mechanisms, but literature has largely not focused on implementation at scale in Pakistan. This is a case report of technical support provided to the Sindh Department of Health/Expanded Program on Immunization (DOH/EPI) from 2015 to 2017 to strengthen routine immunization (RI). The program developed an immunization information system used by district health officers to register and track individual immunization status, improve vaccine logistics, and generate more accurate population estimates and vaccination targets. District immunization officers (DIOs) assisted their district health management teams and supervisors to use registration and service data stored in the immunization information system database to prepare microplans, monitor catchment area performance, and solve problems. Civil society partners registered 830,610 children (aged 0 to 23 months) and 348,315 pregnant women in 28,565 villages over an 18-month intensive intervention period. By the end of this period, $65 \%$ of all registered women had been vaccinated with two or more doses of the tetanus toxoid vaccine (compared to $26 \%$ at baseline); Penta 3 vaccination coverage had increased from $27 \%$ to $64 \%$; and $52 \%$ of the registered children (aged 0 to 23 months) were fully immunized (compared to $18 \%$ at baseline). The immunization information system helped district managers identify and focus limited resources on high-risk populations; reminded families and health providers when vaccinations were due or missed; assisted managers in monitoring vaccination coverage, vaccinator performance, and vaccine stocks; and encouraged local problem solving to improve RI performance. The Government of Sindh demonstrated a commitment to RI based on the program's results, which bode well to future enhancements and scale up.
\end{abstract}

Keywords: Immunization; Pakistan; immunization information system; vaccination; case report

Received: 26 January 2019; Accepted: 07 January 2020; Published: 05 July 2020.

doi: $10.21037 /$ mhealth.2020.01.07

View this article at: http://dx.doi.org/10.21037/mhealth.2020.01.07

\section{Introduction}

Vaccination programs are among the most cost-effective health interventions and a proven tool for preventing and eradicating disease (1). The World Health Organization (WHO) estimates that routine immunization (RI) averts two to three million deaths a year globally and that an additional 1.5 million deaths could be avoided through improved vaccination coverage (2). With the sixth largest population in the world (3), Pakistan ranks third among all countries in terms of the number of unvaccinated and under-vaccinated children (4). Only $54 \%$ of children aged 12-23 months in Pakistan were fully vaccinated at the time of the 2013 Demographic Health Survey (5). WHO estimates that of the 3.8 million infants in the Eastern Mediterranean Region who did not receive the third dose of Pentavalent vaccine in 2015 , approximately $40 \%$ (or 1.52 million infants) were in Pakistan (4).

There are very large unvaccinated urban and rural 
populations nationally and in provinces such as Sindh, in southeast Pakistan. Routine vaccination coverage in Sindh is extremely low: $29 \%$ for the province as a whole and $14 \%$ in rural areas, according to the Pakistan Demographic and Health Survey (PDHS) 2012/2013 (5). Sindh houses some of Pakistan's largest slums, and its capital city, Karachi, is believed to have the largest number of unvaccinated children of any city in the world (6). Because of its low immunization coverage, the province has frequent outbreaks of vaccine-preventable diseases including polio and measles. From 2015 to 2017, there were a total of 22 confirmed cases of polio (7) and over 4,796 cases of measles in Sindh province alone (Government of Pakistan and World Health Organization, 2015-2017, unpublished data).

Among the factors that have had a negative impact on Pakistan's vaccination coverage are outdated census estimates, which make it difficult to set and achieve realistic immunization targets; incomplete and inaccurate vaccination reports; poor worker motivation and supervision of vaccinators; and missed opportunities to link polio eradication and RI activities (8). The authors worked with the Sindh Department of Health/Expanded Program on Immunization (DOH/EPI) from late 2015 through 2017 to strengthen the provincial immunization program. The program of work was part of a larger activity to improve the health of women, newborns, and children in Sindh province by increasing their access to and utilization of key health services, including but not limited to immunization.

This paper describes the implementation of an electronic immunization information system in Sindh province. This paper will be useful to future research and implementation endeavours in the fields of immunization and digital health as it highlights barriers and facilitators, lessons learned, and next steps. Few peer-reviewed publications to-date have focused explicitly on the practical implications of implementation at scale, and several have called for the improved programmatic approaches $(9,10)$. We present the following case in accordance with the CARE Guideline.

\section{Case presentation}

\section{Approach to RI strengthening}

Over an 18-month period, the team worked with a total of 16 districts-eight intensively and eight less intensivelyto improve immunization microplanning, establish more regular vaccination services, provide information to parents and communities about immunization, strengthen the knowledge and capacity of vaccinators and district health managers, and monitor and refine RI service delivery approaches. The program worked with only eight districts intensively due to limitations of both time and resources available from the funder. Districts for intensive work were identified based on larger estimated numbers of unimmunized children relative to the other eight districts where the program was working. Building on an earlier pilot activity that increased RI coverage among children under two years old and pregnant women in four districts of Sindh, the program added new features, including the immunization information system described in this paper.

The approach included training 3,503 vaccinators, district managers, lady health workers, and other types of community health workers on the basics of immunization, creating linkages between vaccinators and local communities, and practical microplanning. The team also mentored district managers during supportive supervision and hired 16 district immunization officers (DIOs) to support the Government of Sindh's district health management teams (DHMTs).

The DIOs in eight of the 16 districts were part of the intensive intervention that included a community microcensus and registration of all pregnant women and children under two years of age by a contracted nongovernmental organization (NGO) network; immunization promotion; assisted microplanning; monitoring of individual and community vaccination status; and other activities. The team developed the immunization information system to store registration data, track individual and community vaccination status, improve EPI logistics, and, over time, generate more accurate population estimates and RI targets. The remainder of this paper focuses specifically on the immunization information system, its characteristics, the authors' experience with it, and the authors' recommendations for future enhancements and use of the system across Sindh province.

\section{Methods for registering women and children}

The immunization information system supported the comprehensive package of technical assistance provided to the Sindh DOH/EPI. To start, field assistants with a local NGO network, the Rural Support Program Network (RSPN), conducted a community micro-censuses and registered all pregnant women and children under two years of age in the 28,565 villages in the eight districts with intensive RI support. Registration data for these women 
and children were collected through paper tools, and then district staff input these data into the electronic system at the facility level. Each registered woman and child was tracked with an individual registration number and, when they were vaccinated, this was recorded in the immunization information system. The system then calculated coverage in terms of the number of pregnant women and children registered and vaccinated by antigen and dose. The DIOs and their government EPI focal points then used the micro-census data to prepare and update immunization microplans. With up-to-date data in hand, district teams were able to more easily complete critical steps in the microplanning process, including estimating the target population for vaccination, determining service delivery strategies, locating immunization outreach sites, and setting immunization targets. Quality microplanning helped them bring vaccination services to remote villages and other underserved populations, maximize the available resources, and improve vaccination coverage and equity.

\section{Data Collection and System Maintenance}

After the initial data collection, continuous follow-up was conducted, and new pregnant women, newborns, and children were added to the registry and database. During each visit by field assistants and vaccinators to the villages, families were reminded about the importance of vaccination and advised about where and when to seek services.

Each week, three computer operators in each district received updated registration sheets from field assistants and entered the data into the immunization information system, either by updating information for existing children and women or by adding new children, women and villages and their information to the database. DIOs used a standard paper checklist when visiting vaccination centers, outreach points, and homes to monitor data quality and collect additional information. DIOs also assisted the DHMTs and District/Taluka supervisors to use registration and service data in monitoring catchment area performance and problem solving.

\section{System description}

The immunization information system comprised of a web portal and integrated mobile messaging platform. The web portal allowed users to store data on individual children and pregnant women; manage, search, and filter client records; and monitor vaccination coverage and vaccinator performance. The portal had a universal login and a set of dashboards to track due doses of vaccines that helped to identify and guide follow-up with defaulters. An integrated mobile messaging platform with web and API access sent short message service (SMS) vaccination reminders and five different awareness messages to caregivers; one reminder for vaccinators to visit specific villages based on their microplans; and one notification to community focal persons informing them that vaccinators would be visiting their villages on certain dates. The cost associated with maintenance of the mobile messaging platform, including the SMS server and messaging costs, was approximately PKR 200,000 or US\$1,700 (11) a month for all intensive intervention districts. Families receiving the text messages did not incur any fees (Figures 1-3).

\section{Methods for building the system}

The technology used to develop the immunization information system module included ASP.NET, C\#, JQuery, and SQL Server. ASP.NET is an open-source server-side web application framework to allow programmers to build websites. C\# is an object-oriented programming language for networking and web development. JQuery is an opensource software designed to simplify the client-side scripting of HTML. Structured query language (SQL) is a relational database management system to store and retrieve data as requested by other software applications.

\section{Discussion}

Electronic census-based immunization information systems have the potential to bolster traditional immunization service delivery mechanisms, but literature has largely not focused on implementation at scale in Pakistan $(9-10,12,13)$. Furthermore, the country has faced challenges in achieving optimal immunization coverage for vaccine-preventable diseases (14) and in sustaining vaccination timeliness (15).

The immunization information system described in this case report was an important ingredient in the program's success in Sindh province. Storing and making population data and client-level information and tools available to the DHMTs and DOH/EPI strengthened program operations and contributed to improvements in vaccination rates. Between March 2016 and August 2017, the program registered 830,610 children and 348,315 pregnant women in the eight intensive intervention districts and entered their data into the electronic immunization information system. 


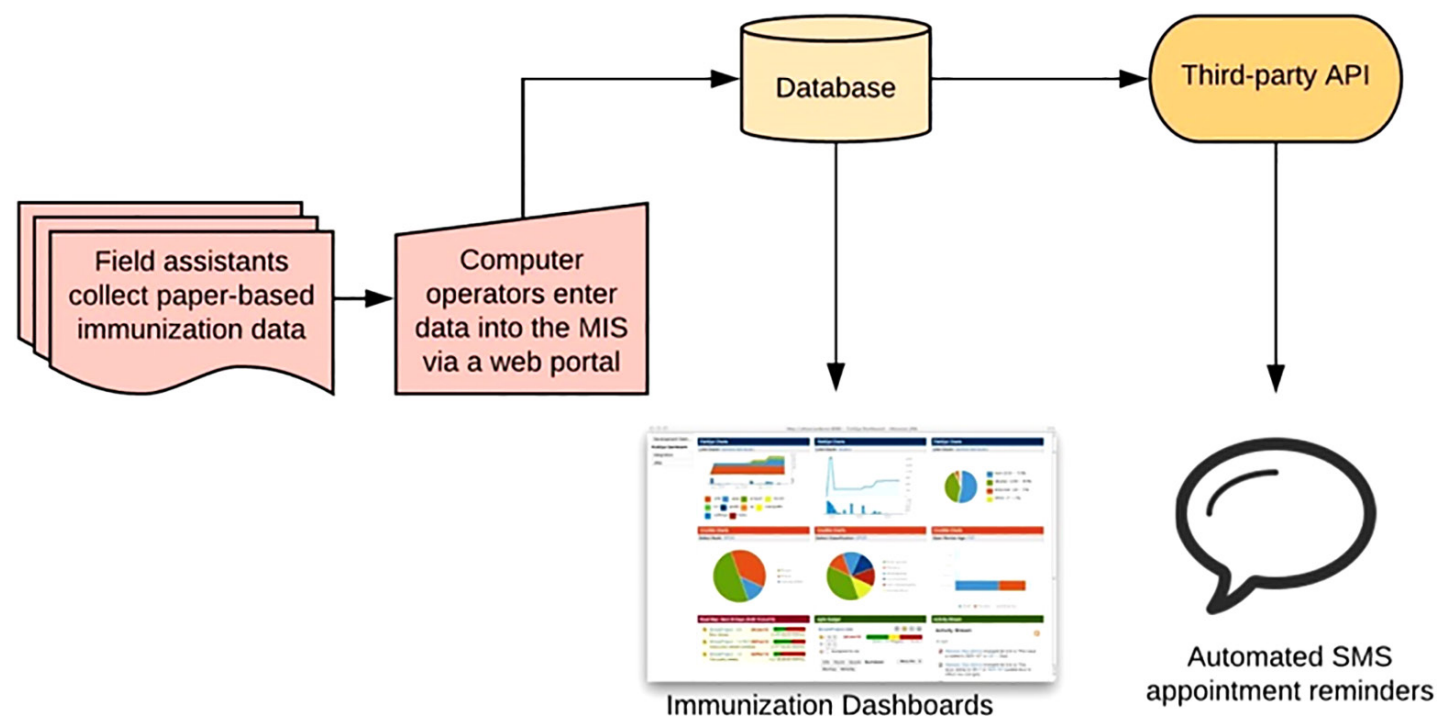

Figure 1 Sindh Immunization Management Information System Workflow.

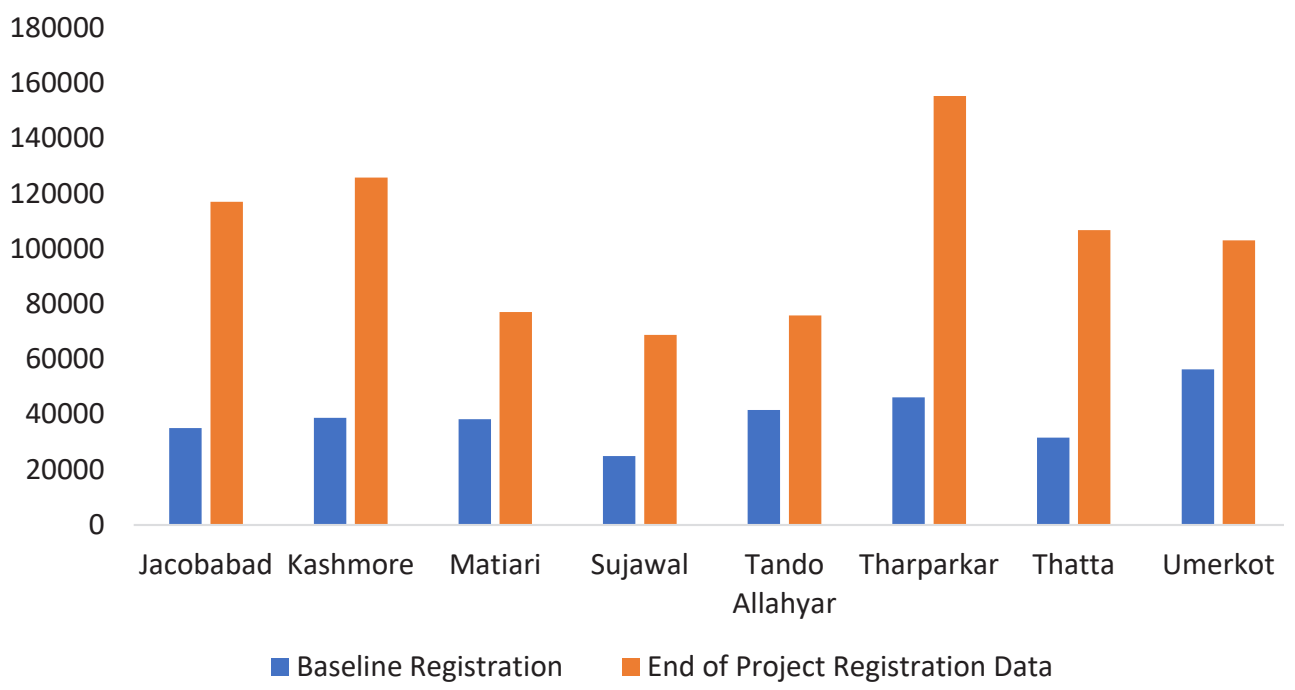

Figure 2 Number of children registered in 8 intensive intervention districts in Sindh Province at baseline and endline (USAID, 2018, unpublished data). In the eight intensive districts, 313,177 children were registered at baseline, and 830,610 children were registered at endline.

By the end of the 18-month intensive intervention period, there was a significant increase in routine vaccination coverage:

(I) About $65 \%$ of all registered women had been vaccinated with at least two doses of tetanus toxoid vaccine, compared to only $26 \%$ at the time of the initial micro-census;

(II) About $64 \%$ of all registered children under two years of age had received a third dose of Pentavalent vaccine, compared to only $27 \%$ at baseline;

(III) About $52 \%$ of registered children were fully immunized, compared to only $18 \%$ at the time of the initial micro-census.

The team began transitioning key elements of the approach, including the immunization information system, to the Sindh Department of Health in late 2017. As part 


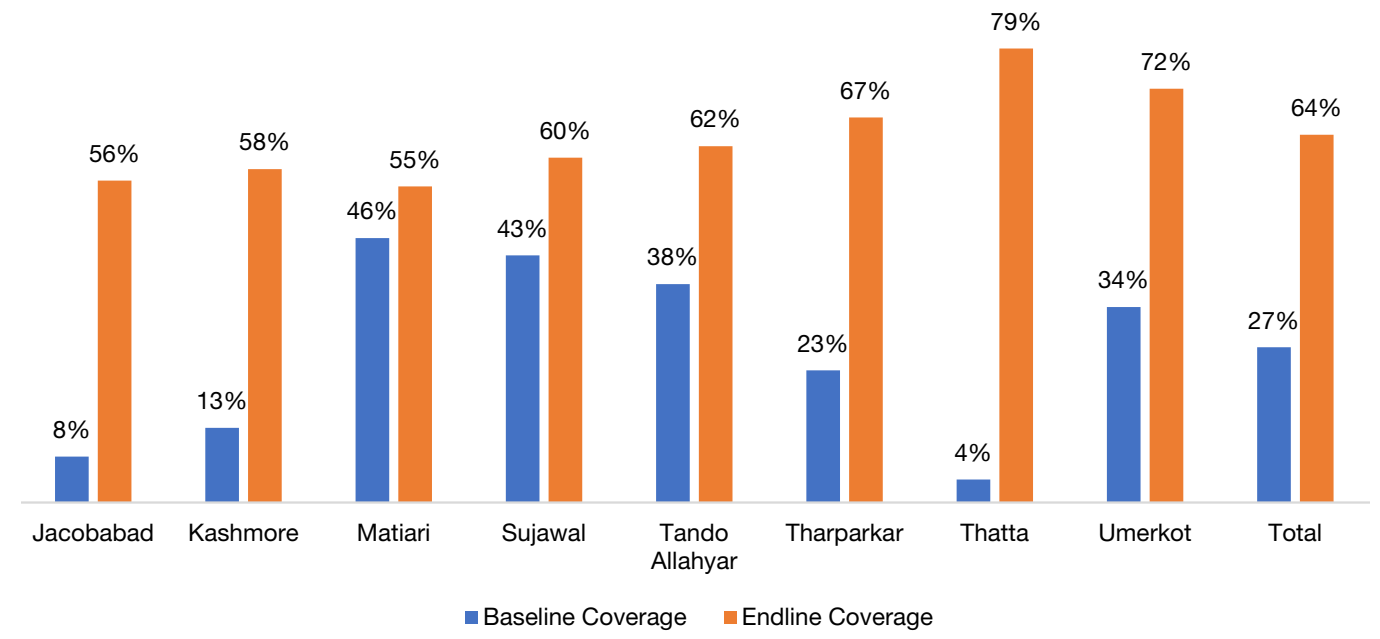

Figure 3 Baseline and endline of project Penta 3 coverage rates in 8 intensive intervention districts in Sindh Province (registered children) (USAID, 2018, unpublished data).

of the transition, plans were to create a single digital immunization information system for immunization by merging the immunization information system described here with the "ZindagiMehfooz" ("Safe Life") mobile application that was being tested in Sindh province with Gavi support and the Government's vaccine Logistics Management Information System (vLMIS), which was managed across the country by the Procurement and Supply Chain Management (PSM) project.

An immunization information system of this kindthat begins with a micro-census of pregnant women and children eligible for vaccination and then tracks these populations over time as part of routine electronic data entry - can facilitate continuous identification of and action to reach children missed by RI efforts. In this case, data generated from the immunization information system was incorporated into district- and provincial-level data review meetings, and these data visualizations catalyzed discussions around performance gaps and how to address them.

In addition to tracking the individual vaccination status of pregnant women and children under the age of two, an immunization information system of this kind could be used by supervisors to hold vaccinators accountable for adhering to their RI outreach session plans. Furthermore, data generated through the system could be used as the basis for meaningful interaction between supervisors and vaccinators around problem-solving to reach missed pregnant women and children under the age of two.

The immunization information system described in this paper required an upfront investment to find and register the initial target population through a community house-to-house micro-census. Tracking and updating the vaccination status of the target population began on paper, and data were entered into the web-based database at the facility level. In the future, the Sindh Department of Health plans to incorporate key elements of the approach described in this manuscript to a single digital information system for immunization. The new system will include a digital application that will make it possible for vaccinators to directly register and track the immunization status of pregnant women and children under two years of age on mobile devices. Vaccinators and their supervisors will be trained to keep this new, fully automated digital information system up-to-date and use the data it produces to develop and adjust their individual immunization session plans, as needed, to improve RI performance.

This program learned that an immunization information system with the capability of sending SMS vaccination reminders and awareness messages to caregivers, reminders to vaccinators to visit specific villages, and notifications to community focal persons informing them of vaccinator visits to their villages has the potential to become an important component of community-based demand generation for vaccination.

This case report is not without limitations. The program did not aim to scientifically assess the effectiveness of the immunization information system, and causation of improved vaccination coverage or timeliness cannot be 
claimed. Future research and literature should build upon this work through randomization, comparing an electronic system to a traditional system. Future literature could also focus on health system barriers and their influencers in Pakistan. A lack of up front program financing, sufficient human resources, adequate supplies and vaccines, and low community awareness and misperceptions surrounding vaccination could negatively affect scale up, sustainability, and replication elsewhere (10).

\section{Conclusions}

The current immunization information system is electronic from the district to the provincial level but paper-based from the household to the district level. Once the combined Sindh immunization information system is fully digitized, it should allow vaccinators and the Sindh DOH/EPI to register and track the vaccination status of individual women and children and monitor vaccine usage and stock levels. Expectations are that it will also auto-generate and send reminder messages to store managers before vaccine expiration dates and to families when new vaccine doses are due or a vaccination is missed. The merging of these three systems is on-going at the time of this writing.

Other important changes that are being made by the Government of Sindh/DOH/EPI, based on the program's results include:

(I) As of February 2018, 77 DHMT members in the eight intensive intervention districts have been trained to register the target population;

(II) The Government of Sindh/DOH/EPI has adopted the practice of hiring and posting DIOs in every district and is recruiting new DIOs for all 29 districts;

(III) The Government of Sindh DOH/EPI has increased its transport allowance for vaccinators from zero to 4,000 Pakistani rupees (US\$25.91) (11) per vaccinator per month beginning in September 2017 , in order to improve resources for RI outreaches.

These policy decisions demonstrate the government's commitment to RI and bode well for future enhancements to the immunization information system.

In rural Sindh, the immunization information system helped district managers identify and focus their limited resources on reaching unimmunized and under-immunized populations with routine vaccination services; reminded families and health providers when vaccinations were missed and encouraged them to take action; assisted managers in monitoring vaccination coverage, vaccinator performance, and vaccine stocks; and encouraged local problem solving to improve RI performance. To make immunization data immediately available for management decisionmaking, future data entry should be electronic at the community and health facility levels, and the immunization information system must be fully owned and operated by the Government of Sindh DOH/EPI.

\section{Acknowledgments}

This publication was made possible through support provided by the United States Agency for International Development (USAID)/Pakistan's Maternal and Child Health $(\mathrm{MCH})$ Program. The opinions expressed herein are those of the authors and do not necessarily reflect the views of USAID.

Funding: None.

\section{Footnote}

Conflicts of Interest: All authors have completed the ICMJE uniform disclosure form (available at http://dx.doi. org/10.21037/mhealth.2020.01.07). ES reports grants from USAID, during the conduct of the study. TM reports grants from USAID, during the conduct of the study. WJ reports grants from USAID, during the conduct of the study. KB reports grants from United States Agency for International Development, during the conduct of the study.SO reports grants from USAID, during the conduct of the study. PR reports other from USAID, during the conduct of the study. SMAG reports other from USAID, during the conduct of the study.

Ethical Statement: The authors are accountable for all aspects of the work in ensuring that questions related to the accuracy or integrity of any part of the work are appropriately investigated and resolved.

Open Access Statement: This is an Open Access article distributed in accordance with the Creative Commons Attribution-NonCommercial-NoDerivs 4.0 International License (CC BY-NC-ND 4.0), which permits the noncommercial replication and distribution of the article with the strict proviso that no changes or edits are made and the original work is properly cited (including links to both the formal publication through the relevant DOI and the license). 
See: https://creativecommons.org/licenses/by-nc-nd/4.0/.

\section{References}

1. Coffield AB, Maciosek MV, McGinnis JM, et al. Priorities among recommended clinical preventive services. Am J Prev Med 2001;21:1-9.

2. WHO. Immunization coverage. Accessed September 4, 2018. Available online: http://www.who.int/mediacentre/ factsheets/fs378/en/

3. 2017 World Population Data Sheet. Accessed June 11, 2018. Available online: https://www.prb.org/wp-content/ uploads/2017/08/WPDS-2017.pdf

4. WHO. Pakistan, Expanded Program on Immunization. Accessed June 11, 2018. Available online: http://www. emro.who.int/pak/programmes/expanded-programme-onimmunization.html

5. Pakistan Demographic and Health Survey, 2012-2013. National Institute of Population Studies. 2013.

6. Progress and Challenges with Achieving Universal Immunization Coverage: 2016 Estimates of Immunization Coverage. UNICEF and World Health Organization. 2017.

7. Polio cases in provinces. Accessed March 21, 2018. Available online: http://www.endpolio.com.pk/polioinpakistan/polio-cases-in-provinces

8. Masud T, Navaratne KV. The Expanded Program on Immunization in Pakistan: Recommendations for improving performance. Accessed September 24, 2019. Available online: https://openknowledge.worldbank.org/ bitstream/handle/10986/13579/NonAsciiFileName0. pdf? sequence $=1$ \& isAllowed $=y$

doi: $10.21037 /$ mhealth.2020.01.07

Cite this article as: Sullivan E, Masood T, Javed W, Bagshaw K, Ollis S, Regmi P, Gardezi SMA. Electronic immunization information systems: a case report of lessons learned from implementation in Pakistan. mHealth 2020;6:31.
9. Shaikh BT, Alizai AA. Head on with the challenges of routine immunization in Pakistan: the need and importance of health systems research. Pakistan J Public Health 2012;2:4-9.

10. Shaikh BT, Haq ZU, Tran N, et al. Health system barriers and levers in implementation of the Expanded Program on Immunization (EPI) in Pakistan: an evidence informed situation analysis. Public Health Rev 2018;39:24.

11. Bloomberg.com. USD to PKR exchange rate. Accessed May 30, 2018. Available online: https://www.bloomberg. com/quote/USDPKR:CUR

12. Scott C, Clarke K, Grevendonk J, et al. Country Immunization Information System Assessments Kenya, 2015 and Ghana, 2016. Morb Mortal Wkly Rep 2017;66:1226-9.

13. Hu Y, Chen Y. Evaluating Childhood Vaccination Coverage of NIP Vaccines: Coverage Survey versus Zhejiang Provincial Immunization Information System. Int J Environ Res Public Health 2017. doi: 10.3390/ ijerph14070758.

14. Chandir S, Dharma VK, Siddiqi DA, et al. Feasibility of using global system for mobile communication (GSM)based tracking for vaccinators to improve oral poliomyelitis vaccine campaign coverage in rural Pakistan. Vaccine 2017;35:5037-42.

15. Noh JW, Kim YM, Akram N, et al. Determinants of timeliness in early childhood vaccination among mothers with vaccination cards in Sindh province, Pakistan: a secondary analysis of cross-sectional survey data. BMJ Open 2019;9:e028922. 